\title{
Systèmes de transformations et actions extérieures $\left({ }^{*}\right)$
}

\author{
P. DELATTRE (**) \\ (Manuscrit rę̧u le 24 février 1976)
}

\begin{abstract}
RÉSUMÉ
On trouvera ici une récapitulation des résultats théoriques et des données expérimentales montrant la nécessité de séparer explicitement le paramètre Intensité du rayonnement et la variable Temps, si l'on veut parvenir à des modèles explicatifs dans l'étude des effets des rayonnements, et si l'on veut que ces modèles permettent l'extrapolation. Cette nécessité devient d'autant plus impérative que l'on considère des rayonnements de plus faible intensité. On montre, également, que dans ce domaine des faibles intensités les phénomènes seront, en général, beaucoup plus complexes qu'aux fortes intensités et, en particulier, que l'on rencontrera souvent des effets non monotones, à dose constante et en fonction du débit de dose. Enfin, dans les systèmes non linéaires fonctionnant en régime périodique stable, un paramètre supplémentaire doit être pris en considération : la phase (moment d'application du rayonnement par rapport au déroulement du cycle de fonctionnement du système étudié).
\end{abstract}

\begin{abstract}
A recapitulation is made of theoretical results and experimental data showing the need to explicitly separate the parameter Radiation intensity and the variable Time if explanatory models are to be obtained in the study of radiation effects, and if these models are to allow extrapolation. This requirement is all the more urgent as the radiations considered are of low intensity. It is also shown that in this low-intensity region the phenomena will generally be much more complex than at high intensities and, in particular non-monotonous effects will often appear at constant dose, as a function of dose rate. Finally in non-linear systems operating under stable periodic conditions an extra parameter must be taken into consideration: the phase (moment of application of the radiation with respect to the development of the working cycle of the system studied).
\end{abstract}

(*) Communication présentée au Séminaire de radiobiologie, organisé par la Société Française de Radioprotection à Fontenay-aux-Roses, les 29-30 avril 1975.

$\left({ }^{* *}\right)$ Commissariat à l'Énergie Atomique, Département de Biologie (G.E.E.R.S.M.), CEN-Saclay, Bât. 28, B. P. n² 2, 91190 Gif-sur-Yvette.

RADIOPROTECTION, VOL. $11-\mathrm{N}^{\circ} 2$ 


\section{I. - INTRODUCTION}

Les effets des rayonnements sur les systèmes chimiques ou biologiques, tels qu'on les conçoit en radiobiologie ou en radioprotection, ne constituent qu'un cas particulier du problème général des actions extérieures sur les systèmes de transformations. Dans la mesure où un cadre conceptuel et théorique précis peut être développé pour le traitement de ces situations générales, on doit s'attendre à ce que les disciplines particulières susceptibles de s'y rattacher en tirent quelque bénéfice. En effet, une théorie ne peut présenter un certain caractère de généralité que si elle est fondée sur un choix pertinent, c'est-à-dire épistémologiquement cohérent, des variables et paramètres de référence auxquels peuvent être rapportés les phénomènes observés; elle doit permettre, en outre, de situer les données que fournit l'expérience, par rapport aux états du système tels qu'ils sont définis dans le cadre théorique choisi. Une théorie générale qui obéit à ces critères constitue une sorte de méta-modèle pouvant servir de base à la comparaison, et donc à l'explication des différents modèles particuliers propres à telle ou telle situation expérimentale.

Pour justifier une approche de ce genre, il est nécessaire de montrer, d'abord, que les questions liées à l'étude des effets des rayonnements se rattachent bien à l'optique des systèmes de transformations. Une fois ce point acquis, nous examinerons quelques-unes des conséquences pratiques qu'entraînent les résultats de la théorie dans le domaine qui nous intéresse ici. Étant donné la brièveté de l'exposé, il ne sera évidemment pas possible d'entrer beaucoup dans les détails, et encore moins de fournir des démonstrations. Je me contenterai donc d'énoncer quelques résultats essentiels en renvoyant, pour des explications plus précises, à des publications antérieures ou à venir.

Rappelons, d'abord, qu'on se trouve en présence de systèmes de transformations chaque fois que l'on étudie des ensembles d'éléments dont les caractéristiques (ou au moins certaines d'entre elles) sont susceptibles de varier au cours du temps, par suite des interactions qui s'exercent entre ces éléments, ou par suite des actions que subissent ces éléments à partir de l'extérieur du système considéré.

On voit immédiatement que cette situation générale peut être rencontrée dans les domaines les plus divers. $\mathrm{Si}$, à partir de là, on précise quels sont les types fondamentaux des transformations possibles (spontanées, dépendant d'un champ extérieur, dépendant d'interactions entre plusieurs éléments), une théorie générale peut être développée [1,2], puis ensuite appliquée à cette catégorie particulière de phénomènes que sont les effets des rayonnements [3-5]. Je ne reviendrai pas ici sur le contenu précis de cette théorie, qui a déjà été exposée à plusieurs reprises. Mais il me semble utile de retenir surtout que la plupart des conclusions qualitatives tirées de la théorie et, parmi celles-ci, celles dont il sera question plus loin, ne résultent en définitive que des considérations suivantes sur lesquelles est fondée la théorie :

a) Les phénomènes observés expérimentalement se rattachent à des états différents des objets étudiés. 
b) Une propriété mise en évidence expérimentalement est, en général, liée à certains états, et non à la totalité des états, que peuvent occuper les éléments du système étudié.

c) Entre les états observés (catégories d'identification expérimentale) et les états réels des objets dans le système considéré (classes d'équivalence), on ne peut pas affirmer qu'il y a toujours correspondance biunivoque; en général, à un état observé correspondent plusieurs états réels possibles, ou inversement $(*)$.

d) Au cours du temps, le nombre d'objets dans un état réel donné varie. Le nombre de transformations par unité de temps entre deux états quelconques peut dépendre :

- des taux de transformations spontanées, liés aux propriétés intrinsèques des objets; de l'intensité du rayonnement appliqué (cas linéaires);

- des taux d'interactions pour les différentes transformations mettant en jeu plusieurs objets identiques ou dissemblables (cas non linéaires).

On constatera que ces différentes affirmations ne sont pas à proprement parler des hypothèses, mais qu'elles expriment plutôt soit des constatations immédiates, voire évidentes (points $a, b, c$ ), soit des exigences liées à la cohérence interne du formalisme (point $d$ ). De là, résultent le haut degré de généralité et l'étendue du domaine de validité des conclusions que l'on peut en déduire.

\section{II. - LA VARIABLE TEMPS ET LE PARAMÈTRE INTENSITÉ}

Parmi les résultats qu'a permis d'obtenir l'application de la théorie des systèmes de transformations à l'étude des effets des rayonnements, l'un des plus fondamentaux me semble être celui qui concerne la nécessaire séparation de la variable Temps et du paramètre Intensité du rayonnement, si l'on veut parvenir à l'élaboration de modèles comparables entre eux et extrapolables. Bien que j'aie l'impression d'avoir déjà beaucoup rabâché cette question, oralement et par écrit, il n'est sans doute pas inutile d'y revenir car la démonstration que j'ai donnée ne semble pas avoir été très bien comprise, dans ses implications fondamentales.

Certains ont trouvé cette démonstration difficile, à cause du formalisme mathématique qu'elle utilise; d'autres n'y ont vu, précisément, qu'un développement mathématique sans intérêt pratique; d'autres, enfin, ont estimé qu'elle n'apportait aucun élément nouveau.

Il n'y a pas grand chose à dire de la première critique, si ce n'est qu'elle manifeste la nécessité, d'une part d'un effort pédagogique, d'autre part d'un effort de compréhension qui implique l'acquisition de certaines connaissances mathématiques, à vrai dire assez simples.

La seconde critique est, évidemment, une erreur manifeste. Quiconque veut bien examiner avec quelque attention les bases de la théorie, et la procédure

(*) Ceci résulte simplement du fait que les propriétés mises en jeu dans l'identification expérimentale des objets sont, en général, différentes de celles que manifestent ces mêmes objets dans le système que l'on étudie.

voL. $11-\mathrm{N}^{\circ} 2$ 
de recherche des modèles qui lui est associée $[6,7]$ s'en rend compte très vite. En réalité, cette théorie est étroitement liée aux procédures expérimentales effectivement mises en œuvre ( $c f$., en particulier, $\S \mathrm{I}, a, b, c)$ et présente, de ce fait, une valeur empirique très supérieure à celle des modèles a priori généralement utilisés.

La troisième critique, en revanche, touche un point essentiel, et l'on peut même dire que c'est autour de cette question que se situent les véritables difficultés de compréhension. Il est bien certain que les radiobiologistes et les spécialistes de la Radioprotection ont depuis longtemps reconnu que l'intensité $\Phi$ du rayonnement (ou, si l'on préfère, le débit de dose $d$ ) joue un certain rôle dans la réponse de nombreux systèmes. Mais, pour tenir compte de ce fait, ils ont estimé qu'il suffisait de considérer le débit de dose comme un simple facteur correctif venant parfois perturber les réponses en fonction de la dose, considérée comme référence fondamentale. C'est précisément cette manière d'opérer qui, à mon avis, est très sujette à caution, tout au moins si l'on veut que le formalisme utilisé pour la modélisation se prête aux extrapolations et aux prévisions les plus élémentaires, celles qui concernent justement les effets d'intensité.

En effet, considérer que le débit de dose n'est qu'un facteur correctif c'est l'exclure de la théorie, c'est en faire un simple appendice facultatif et occasionnel, non relié aux aspects fondamentaux des phénomènes. Certains auteurs ont bien tenté de surajouter, à des modèles exprimés en fonction de la Dose, des termes dépendant du débit de dose ou du temps, mais je ne connais pas de modèles de ce genre qui aient donné des résultats satisfaisants. Ceci est tout à fait compréhensible puisque le temps intervient alors, d'une part comme variable séparée explicite, et d'autre part comme variable implicite dans la Dose, ce qui est évidemment peu recommandable du point de vue théorique. Le moins que l'on puisse dire est que cette manière de faire complique beaucoup et inutilement les choses.

Pour important qu'il soit, cet argument formel n'est pas, et de loin, le seul à jouer en faveur d'une séparation systématique de la variable Temps et du paramètre Intensité. En me référant aux publications antérieures [3, 4], je rappellerai que dans tout système où se produit au moins une transformation irréversible, ce qui est le cas le plus fréquent, l'addition dans la Dose de tous les dépôts d'énergie revient à confondre des phénomènes-causes et des phénomènes-effets, ce qui ne peut que conduire à de nombreuses difficultés d'interprétation. On peut également montrer [3,4] que cette procédure entraîne, en général, l'addition arithmétique de grandeurs énergétiques ayant, pour l'évolution du système, des significations qualitatives différentes, ce qui n'est pas non plus très orthodoxe.

Il est bon de se souvenir aussi que l'introduction du concept de Dose comme référence pour l'étude des effets des rayonnements date des premières recherches sur les rayons $\mathrm{X}$, à la fin du $\mathrm{XIX}^{\mathrm{e}}$ et au début du $\mathrm{XX}^{\mathrm{e}}$ siècle $\left(^{*}\right)$, c'est-à-dire

(*) Cet usage de la notion de Dose doit même, me semble-t-il, être rattaché à la loi de réciprocité de Bunsen et Roscoe, énoncée en 1862 à propos de la photochimie des émulsions photographiques. 
d'une époque où l'on prêtait à la thermodynamique un caractère de généralité très supérieur à celui qu'elle possède en réalité. C'était l'époque où l'on espérait encore la mise au point d'une théorie énergétiste globale (RANKINE, OsTWALD), capable d'effectuer la synthèse des domaines scientifiques les plus divers. Dans ce contexte, il était assez naturel de voir naître et se développer une dosimétrie des rayonnements, fondée elle aussi sur le concept global d'énergie reçue ou cédée par un système. On sait maintenant que ce concept global ne peut guère servir de référence qu'au voisinage des états d'équilibre thermodynamique, et pour l'étude de grandeurs macroscopiques, elles aussi globales, c'est-à-dire concernant, en tant que moyennes, tous les éléments d'un système (pression, volume, etc.). On sait aussi que la plupart des phénomènes relatifs aux effets des rayonnements n'obéissent à aucun de ces deux critères : les systèmes biologiques vivants travaillent en général loin des états d'équilibre thermodynamique; et les propriétés que l'on étudie ne concernent pas, en général, la totalité des éléments du système mais seulement certains des états que peuvent occuper ceux-ci $(c f . \S \mathrm{I} b)$. Dans ce cas, on ne peut échapper à la nécessité des études de dynamique des systèmes, c'est-à-dire de cinétique des transformations, qui implique obligatoirement l'introduction du temps comme variable de référence explicite. On remarquera d'ailleurs, à ce sujet, que même les théories dites de " thermodynamique irréversible " n'échappent pas à cette nécessité dès qu'il s'agit d'étudier les comportements loin de l'équilibre thermodynamique (structures dissipatives de PRIGOGINE).

Un autre argument en faveur de la prise en compte explicite des facteurs Temps et Intensité résulte directement de ce que nous savons concernant la nature des transformations qui se produisent effectivement dans un système irradié. C'est aujourd'hui un lieu commun de rappeler que certaines de ces transformations sont provoquées par le rayonnement, alors que d'autres n'en dépendent pas, c'est-à-dire se déroulent selon leur cinétique propre. Pour rendre compte des changements que subit un système quelconque il est donc, d'une manière tout à fait générale, indispensable de traiter conjointement ces deux types de phénomènes puisqu'ils interviennent simultanément. Et ceci n'est possible que si on les exprime tous deux en termes comparables, c'est-à-dire en taux de transformations par unité de temps. De là résulte la nécessité d'introduire le facteur Intensité (ou le débit de dose) auquel sont reliés directement les taux des transformations dépendant du rayonnement, ainsi, bien entendu, que le temps dont dépendent les transformations spontanées et qui sert aussi de référence à la cinétique d'ensemble du système.

Il va de soi que si l'on considère seulement les effets des rayonnements de très forte intensité, c'est-à-dire si l'on travaille dans un domaine où les transformations dues au rayonnement sont beaucoup plus rapides que les transformations spontanées, il devient possible d'admettre un traitement de l'évolution du système en phases successives : sous rayonnement, où l'on admettra que les transformations du premier type interviennent seules et où la notion de Dose peut donc être employée [5]; et hors rayonnement, où n'interviennent que celles du second type. Mais il est bon de ne pas perdre de vue que cette manière d'opérer n'est qu'une approximation, qui devient de moins en moins justifiée au fur et à mesure que l'on considère des rayon-

voL. $11-\mathrm{N}^{\circ} 2$ 
nements d'intensités plus basses. Les deux types de phénomènes deviennent alors aussi fondamentaux l'un que l'autre, et l'idée de considérer les seconds comme facteurs correctifs des premiers perd pratiquement toute signification. Cette situation apparaît d'ailleurs très clairement lorsque l'on constate que le signe de variation de certains effets peut changer selon les gammes d'intensité considérées (effets non monotones) [8,9], ce qui ne fait que traduire, en définitive, une compétition entre les deux types de transformations. Il devient alors difficile d'admettre que de simples facteurs correctifs puissent rendre compte de ces phénomènes. Mais quelques explications complémentaires sont, sans doute, nécessaires sur ce point.

L'existence d'un effet non monotone se traduira, en principe, par une courbe où, pour une dose donnée, la variation de l'effet en fonction de l'intensité du rayonnement présente un extremum (maximal ou minimal) pour une certaine intensité.

On peut montrer que l'existence de tels effets est possible aux basses intensités dans des systèmes de transformations très variés. Il semble même, dans l'état actuel des études, que les conditions de non-existence de ce type d'effet soient nettement plus restrictives que les conditions d'existence [10]. Autrement dit, il semble que l'on doive s'attendre à rencontrer ces effets non monotones dans de nombreux systèmes soumis à de basses intensités de rayonnements.

L'intérêt de ce résultat théorique n'est, sans doute, pas seulement d'ordre spéculatif puisque certains résultats expérimentaux semblent le confirmer $[8,11,12]$. Par exemple, dans des travaux récents, W. P. NorRIS et T. E. FriTz ont montré, sur des chiens beagle soumis à une irradiation chronique par les gammas du cobalt 60 , que la gravité de l'effet, pour une dose totale donnée, augmente lorsque le débit de dose diminue, dans la gamme de 5 à $35 \mathrm{R} / \mathrm{jour}$ $[11,12]$.

On voit, en définitive, que les arguments en faveur d'une séparation explicite des facteurs Temps et Intensité, dans les modèles théoriques qui se veulent explicatifs, sont nombreux et, me semble-t-il, convenablement étayés du point de vue scientifique. Pour terminer l'examen de ce point, il n'est sans doute pas inutile de se demander ce qui, à l'heure actuelle, peut s'opposer à ce changement d'optique, apparemment facile et en tout cas indispensable pour l'étude des effets des rayonnements de basse intensité. Sans prétendre épuiser cette question délicate dont les implications ne sont probablement pas toutes d'ordre scientifique, je m'arrêterai aux trois causes suivantes qui me paraissent les plus fortes :

- la difficulté d'abandonner des habitudes de pensée anciennes qui ont fini par constituer un cadre de réflexion commode;

- le fait que l'étude des effets des rayonnements ait surtout, jusqu'à présent, concerné des rayonnements d'intensité élevée, pour lesquels la notion de dose constitue une référence justifiée, à titre d'approximation;

- le fait qu'il est certainement plus facile de mesurer des quantités d'énergie globale déposée plutôt que des débits de dose en fonction du temps. 
Pour compréhensibles que soient ces obstacles à un changement d'optique, il n'en reste pas moins nécessaire de s'efforcer de les lever si l'on veut parvenir à une compréhension convenable des effets des rayonnements de faible intensité.

\section{III. - LES CHANGEMENTS D'ÉQUILIBRES CINÉTIQUES}

Dans ce qui précède, nous n'avons considéré que des effets dont l'importance quantitative peut varier, éventuellement de manière non monotone, en fonction de l'intensité (ou débit de dose) du rayonnement, pour une même dose et pour un même type de rayonnement, mais dont la nature qualitative n'est pas modifiée par ces variations d'intensité. Cette situation relativement simple est loin d'être générale. Il peut arriver que de simples variations d'intensité fassent apparaître des changements qualitatifs des effets observés [9], ce qui entraîne un argument supplémentaire, plus impératif encore que les précédents, pour que le facteur Intensité soit considéré comme un paramètre fondamental et non comme un simple terme correctif.

Je voudrais essayer de montrer, brièvement, comment se présente cette question. Il va de soi qu'entre l'absence complète et la présence d'un rayonnement donné, des variations qualitatives interviennent généralement dans les systèmes considérés, puisque le rayonnement entraîne, pour les éléments du système, la possibilité d'états nouveaux qui n'existaient pas auparavant. Ces variations qualitatives peuvent, cependant, n'avoir qu'une importance négligeable $(*)$, en particulier si le rayonnement appliqué est d'intensité suffisamment voisine de 0 , ce qui revient à dire que les états nouveaux possibles pour les éléments ne sont occupés par ceux-ci qu'à un taux très faible. Malgré tout son intérêt théorique, ce problème des conséquences du passage d'une intensité nulle à une intensité non nulle d'un rayonnement n'est sans doute pas le plus important en pratique, ne serait-ce que parce que les systèmes que nous étudions fonctionnent déjà dans un environnement naturel non dénué de rayonnements très divers. Il semble beaucoup plus intéressant de savoir si les variations d'intensité, pour les valeurs non nulles de celle-ci, sont parfois susceptibles de provoquer également des changements qualitatifs du comportement des systèmes.

Il reste à préciser ce que l'on entend, dans cette optique, par « changement qualitatif ". Dans le cadre des systèmes dynamiques, dont font partie les systèmes biologiques, la manière la plus générale de définir cette expression consiste à dire qu'il y aura changement qualitatif chaque fois que seront modifiées les propriétés de stabilité intrinsèque (homéostasie) d'un système, ou d'une partie de celui-ci, vis-à-vis des perturbations occasionnelles qu'il peut subir. Ceci peut concerner aussi bien la disparition d'un état stable (statique ou dynamique), que le passage d'un premier état stable à un second, avec impossibilité d'un retour spontané du second au premier.

(*) Sauf, bien entendu, si les nouveaux états entraînent l'apparition de chaînes catalytiques ou autocatalytiques susceptibles de provoquer des transformations à caractère " explosif ". 
Nous allons voir, maintenant, quelques situations typiques que l'on peut, de ce point de vue, rencontrer dans les systèmes de transformations.

Notons d'abord que tout système biologique, et $a$ fortiori toute partie de système biologique, fonctionne en système ouvert, c'est-à-dire reçoit de l'extérieur et rejette vers l'extérieur certains de ses éléments constitutifs. Les propriétés dont il sera question un peu plus loin concernent donc les systèmes ouverts.

Rappelons également que les variations d'intensité du rayonnement modifient certains taux de transformations des éléments, c'est-à-dire certains taux de changements d'états de ceux-ci, par unité de temps. Si les intensités considérées ne sont pas trop élevées, les transformations irréversibles que peut provoquer le rayonnement ne prendront pas une importance relative prépondérante par rapport aux transformations réversibles (provoquées ou non par le rayonnement); dans ce cas, les systèmes considérés seront, statistiquement, plutôt perturbés que détruits. Ceci n'exclut d'ailleurs pas que ces perturbations puissent être plus ou moins graves pour l'intégrité ultérieure du système, et que certaines d'entre elles puissent provoquer, à plus ou moins long terme, une sorte d'autodestruction différée de celui-ci, par évolution spontanée à partir des nouvelles conditions de fonctionnement. La notion de perturbation utilisée ici, et à laquelle se rattachent les considérations qui vont suivre, concerne en premier lieu les changements qui ne modifient pas la structure fondamentale du système, mais seulement les fonctionnements relatifs ou réciproques des différentes parties de celle-ci. Une telle notion permet donc de distinguer ces changements de ceux qui correspondent à des destructions structurales définitives, sous l'action même du rayonnement, par exemple par suite de la destruction complète de certains éléments non remplaçables. Cette manière d'aborder l'étude des effets des rayonnements est tout à fait dans la ligne de ce qui a été exposé précédemment ( $c f$. $\S$ I et II). Rappelons que son intérêt réside surtout dans le fait qu'elle permet un traitement simultané de la cinétique propre des systèmes et de l'incidence que peuvent avoir sur celle-ci les variations des rayonnements appliqués, les destructions directes provoquées par ces rayonnements n'apparaissant plus alors que comme des cas particuliers dans un ensemble de réponses possibles pouvant être très diverses et plus ou moins complexes.

\section{a) Systèmes fonctionnant sur un point singulier stable [13, 14]}

Dans ce cas, et pour des conditions extérieures fixes, l'occupation des différents états possibles par les éléments constitutifs du système reste constante.

Pour les systèmes de transformations linéaires ouverts les plus simples, c'est-à-dire sans processus multiplicatif, le point d'équilibre se déplace en fonction de l'intensité du rayonnement, mais les différents points d'équilibre atteints pour les différentes valeurs de l'intensité présentent tous le même caractère de stabilité vis-à-vis des perturbations qui peuvent intervenir sur les paramètres ou sur les entrées du système. Cette stabilité est non seulement locale (petites perturbations momentanées), mais aussi globale. Si l'on passe 
d'une intensité $\Phi_{1}$ du rayonnement (point singulier $P_{1}$ ) à une intensité $\Phi_{2}$ (point singulier $P_{2}$ ), le retour de $\Phi_{2}$ à $\Phi_{1}$ ramène le système du point $P_{2}$ au point $P_{1}$.

Si l'on considère des systèmes de transformations linéaires ouverts, avec processus de multiplication, les propriétés précédentes restent valables si le rayonnement ne participe à aucune des transformations multiplicatives. Mais si cette condition restrictive n'est pas remplie, la stabilité du système peut disparaître pour certaines gammes d'intensité du rayonnement. Cependant le retour, par variation de cette intensité, d'une zone d'instabilité à une zone de stabilité ramènera, en général, le système à son état stable antérieur, si toutefois l'excursion instable n'a pas, entre-temps, provoqué l'apparition de nouvelles caractéristiques de fonctionnement.

[On remarquera, au passage, que si l'on considérait des systèmes de transformations linéaires semi-ouverts ne recevant de l'extérieur aucun de leurs éléments constitutifs, le retour à un point d'équilibre initial, après passage par d'autres points, ne serait possible que pour les valeurs relatives d'occupation des différents états par les éléments du système, et non plus pour les valeurs absolues comme c'est le cas avec les systèmes ouverts. Ce résultat traduit simplement le fait que de tels systèmes linéaires semi-ouverts gardent une certaine mémoire de leurs états antérieurs (au niveau des valeurs absolues d'occupation des classes), alors que les systèmes ouverts ont des états d'équilibre totalement indépendants de ces états antérieurs.]

En ce qui concerne les systèmes non linéaires, la situation est généralement plus complexe et, surtout, elle dépend dans une large mesure de la structure particulière des transformations dans le système considéré. Les différences essentielles de comportement, par rapport aux systèmes linéaires, portent sur les aspects suivants. Dans certaines gammes de variations de l'intensité du rayonnement, le système changera d'état d'équilibre quand variera cette intensité, mais avec possibilité de retour dans son état initial lorsque le rayonnement reprendra sa valeur initiale. Pour d'autres gammes d'intensité, le système passera dans de nouveaux domaines de points singuliers stables, ou de situations cycliques stables [ $c f$. point b) ci-dessous], sans possibilité de retour spontané à son état antérieur, même si l'intensité du rayonnement reprend sa valeur initiale. Il est également possible que le système soit entraîné dans des domaines instables, à partir desquels il poursuivra ensuite une évolution spontanée plus ou moins rapide lorsque le rayonnement reprendra son intensité initiale. D'un point de vue général, on ne peut rien dire a priori du sens de cette évolution spontanée, qui peut aussi bien ramener le système dans son état initial que l'en éloigner définitivement.

Il existe une catégorie de systèmes non linéaires, susceptibles de fonctionner sur des points singuliers stables, et qui devraient, en principe, être particulièrement sensibles aux variations paramétriques telles que celles que provoquent les variations d'intensité d'un rayonnement. Il s'agit des systèmes dits "à régulation inversée » $[13,14]$ dans lesquels la quantité d'éléments d'un type donné, dans l'état d'équilibre, diminue lorsque l'apport extérieur de ces mêmes éléments augmente, et inversement. Ces systèmes, qui jouent sans doute

voL. $11-\mathrm{N}^{\circ} 2$ 
un rôle important dans de nombreux processus biologiques, et que l'on rencontre en particulier en endocrinologie, n'ont malheureusement encore été que très peu étudiés. On peut, cependant, démontrer théoriquement qu'ils fonctionnent sur des états stables assez voisins de situations instables; ce résultat laisse supposer qu'ils devraient être relativement sensibles aux variations paramétriques que peut produire un rayonnement incident.

\section{b) Systèmes fonctionnant en régime cyclique stable}

Il s'agit ici des situations du genre cycles-limites, que l'on ne peut rencontrer qu'avec les sytèmes non linéaires. Dans ces situations, le nombre d'éléments dans chaque état possible varie de façon périodique au cours du temps.

Les variations paramétriques liées aux changements d'intensité d'un rayonnement incident pourront, comme dans les cas précédents [ $c f$. § III a], faire passer le système dans de nouveaux états, stables ou instables, spontanément réversibles ou non vers l'état initial. Les nouveaux états stables ne seront pas forcément cycliques [9]. Mais le fait de partir d'un état initial cyclique a pour conséquence qu'au moins une nouvelle variable devra être prise en considération pour rendre compte des phénomènes observés. Outre l'intensité du rayonnement et sa variation dans le temps, il sera nécessaire de tenir compte aussi de sa phase, c'est-à-dire des instants où interviennent ses variations d'intensité par rapport au déroulement du cycle où se trouve engagé le système considéré. On devra s'attendre à trouver des effets différents selon l'époque d'application du rayonnement, tout au moins pour les irradiations relativement brèves par rapport à la période du cycle.

Ce genre de phénomène n'a encore été que peu étudié. Les travaux les plus importants dans ce domaine sont certainement ceux de A. T. WiNFREE [16-18], qui a entrepris depuis quelques années toute une série d'études, à la fois théoriques et expérimentales, du plus grand intérêt. Il a montré, en particulier, qu'une impulsion lumineuse d'intensité et de durée précises, appliquée à un instant précis du cycle circadien de la drosophile, était susceptible de supprimer ce cycle et de faire disparaître, par conséquent, les rythmes physiologiques qu'il commande. Des impulsions identiques appliquées à des instants différents du cycle peuvent être sans effet, ou entraîner des effets intermédiaires tels que le décalage temporel du cycle.

Notons, pour terminer, que les travaux de WINFREE ne sont pas seulement intéressants et instructifs pour l'étude des effets des rayonnements. Ils s'appliquent aussi à d'autres types d'actions extérieures, et en particulier aux variations des apports d'éléments que l'on peut produire en agissant sur les entrées des systèmes ouverts. Certaines de ces études ont été consacrées, par exemple, aux conséquences des variations d'apport d'oxygène dans le système glycolytique de levures, où peuvent apparaître également des effets de phase.

\section{IV. - CONCLUSION}

J'espère avoir montré, par les résultats théoriques et expérimentaux qui viennent d'être décrits, la nécessité de faire évoluer l'étude des effets des 
rayonnements vers des formes plus élaborées que celles qui ont été développées dans le passé.

Si les observations concernant les rayonnements de haute intensité ont pu être décrites, avec une approximation convenable, à l'aide de modèles très simples, la situation devient radicalement différente dès qu'il s'agit de rendre compte des phénomènes provoqués par les rayonnements de basse intensité, qui interfèrent étroitement avec la cinétique propre des systèmes. Il semble que le moment soit venu de passer de la simple dosimétrie des rayonnements à une véritable cinétique des effets des rayonnements, qui n'éludera plus le rôle de la dynamique interne des objets étudiés.

Je crois que l'on risque d'être très surpris, lorsque les études à entreprendre dans cette optique seront plus avancées, par la grande variété, voire la complexité, des effets possibles aux basses intensités. Ceci ne préjuge évidemment en rien de la signification pratique que peuvent avoir ces effets. Tout ce que l'on peut dire à ce sujet, dans l'état actuel des connaissances, c'est qu'il est sans doute dans une large mesure erroné d'extrapoler aux basses intensités les effets observés aux fortes intensités où les réactions propres des systèmes irradiés sont quantitativement " dépassées " par les taux très élevés des transformations provoquées directement par le rayonnement. Seules, les transformations totalement irréversibles, dont il resterait à faire un inventaire précis, pourraient éventuellement justifier une telle extrapolation.

A notre époque où de nombreuses disciplines reconnaissent la nécessité de s'engager dans des études de dynamique fine, où la thermodynamique se voit adjoindre progressivement une théorie cinétique des transformations, où la pharmacologie devient peu à peu une véritable pharmacocinétique, il serait dommage que les spécialistes de la dosimétrie perdent un temps précieux en ne prenant pas conscience assez tôt de la nécessité d'une évolution analogue.

\section{BIBLIOGRAPHIE}

[1] Delattre P. L'évolution des systèmes moléculaires. Paris, Maloine, 1971.

[2] Delattre P. Système, structure, fonction, évolution. Paris, Maloine, 1971.

[3] Delattre P. Étude des fondements de la dosimétrie à la lumière d'une théorie générale des systèmes de transformations. 3rd Symposium on Microdosimetry, Stresa, 1971 (EUR-4810-d-f-e), Communautés européennes, Luxembourg, 1972, 701-737.

[4] Delattre P. Systems theory and radiation research. General systems yearbook, 1973, 18.

[5] Delattre P. Systems approach of theoretical models in radiobiology and radiotherapy. Int. J. General Systems, 1974, 1. 105-117.

[6] Delatrre P. Traitement conjoint des phénomènes dépendant ou ne dépendant pas du rayonnement et recherche des modèles d'interprétation théorique. 4th Symposium on Microdosimetry. Verbania, 1973 (EUR-5122-d-e-f), Communautés européennes, Luxembourg, 1974, 279-313.

[7] Delforge J. Étude sur les problèmes d'identification dans les systèmes de transformations linéaires. Thèse, Orsay, 1975.

[8] Lyon M. F., Papworth D. G., Phillips R. J. S. Dose-rate and mutation frequency after irradiation of mouse spermatogonia. Nature New Biology, 1972, 238, 101-104. 
[9] Hyver C. Effets non monotones en fonction du débit de dose. note interne $\mathrm{n}^{\circ} 41,1972$.

[10] Delattre P. Note sur les conditions d'apparition des effets non monotones aux faibles intensités de rayonnement (à paraître).

[11] Norris W. P., T. E. Fritz. Response of the Beagle dog to protracted exposure to ${ }^{60} \mathrm{Co}$ gamma-rays. 5th International Congress of Radiation Research, Seattle, 1974 (à paraître).

[12] Fritz T. E., Norris W. P. Pathologic responses of the Beagle dog to protracted exposure to ${ }^{60} \mathrm{Co}$ gamma-rays. 5th International Congress of Radiation Research, Seattle, 1974 (à paraître).

[13] DelatrRe P. Systèmes de transformations à caractéristique temporelle et théorie des populations. Note interne $\mathrm{n}^{\circ} 48$, septembre 1974.

[14] Delatrre P. Quelques aspects de la régulation dans les systèmes de transformations. Actes du Colloque du Collège de France sur l'idée de régulation dans les Sciences. Paris, Maloine, 1976.

[15] Bernard-Weil E., Pilleron J. P. Association between vasopressin and corticosteroids in the palliative treatment of advanced breast cancer. Oncology, 1973, 23, 492-508.

[16] Cowan J. D., Levins R., MacArthur R., Winfree A. T., Eds. Proceeding A.A.A.S. Amer. Math. Soc. Symposium, New York, 1967, Dallas, 1968. Amer. Math. Soc. Publication, 1970.

[17] Winfree A. T. Time and timelessness in biological clocks. In Temporal aspect of therapeutics (UrquHart J. and Yates E., eds.) New York, Plenum Publishing Company, 1968, 35-57.

[18] Winfree A. T. Patterns of phase compromise in biological cycle. J. Math., Biol., 1974, 1, 73-95. 